\title{
A Novel Web Service Recommendation Approach based on Credible User Comment
}

\author{
Denghui Wang ${ }^{1, a}$, Hao Huang ${ }^{2, b}$ and Changsheng $\mathrm{Xie}^{3, \mathrm{c}}$ \\ ${ }^{1}$ School of Computer Science and Technology, Huazhong University of Science \& Technology, \\ Wuhan 430074, China \\ ${ }^{2}$ School of Computer Software Engineering, Huazhong University of Science \& Technology, Wuhan \\ 430074, China \\ ${ }^{3}$ Wuhan National Laboratory for Optoelectronics, Huazhong University of Science \& Technology, \\ Wuhan 430074, China

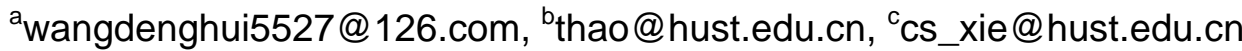

Keywords: user evaluation, trust degree, web service recommendation.

\begin{abstract}
User comment is an important criterion for the approach of web service recommendation. But estimating the credibility of user comment is always a big challenge. This paper proposes a novel method to calculate the trust degree of the user evaluation. In the proposed method, the trust degree of the user evaluation is estimated with difference value derived by comparing the user satisfaction degree with user comment. Then the paper proposes an approach of web service recommendation based on the credible user evaluation. In the final, we present an experiment to prove the new approach which is accurate and effective can avoid the influence of false user comment.
\end{abstract}

\section{Introduction}

With the development of network technology, web services are applied widely. There exist a number of available web services providing similar or identical functional characteristics, so users need a recommendation approach that can help them choose the best web service [4]. Recently more and more researches about web service recommendation are proposed.

User comment can evaluate a web service from multiple aspects. It constitutes the user experience of the web service. However, there is a problem when recommendation system applies user comment to evaluate web service composition, that is, dishonest consumers might reply a false feedback to affect the evaluation results [3]. False user comment issue might cause to raise an improper recommendation due to the lacking of a good trust degree measure scheme. To cope with this problem, it is necessary to avoid false comment by dishonest consumers in the recommendation process. In this paper, a web service recommendation approach, based on reliable user evaluation, is proposed which takes past consumer behaviors.

This paper is organized as follows: in the second sections, we emphatically introduce the related work about web service recommendation. The third section introduces user evaluation model and user requirement model for evaluate user comment. The fourth section explains the method to calculate the trust degree of user evaluation. And then we present a recommendation approach of web service composition that is based on reliable user evaluation. The fifth section proposes the experiment that leads to prove the method is accurate and effective. The sixth section gives the final conclusion.

\section{Related Works}

The QoS is the measurement of the non-functionality attribute of web services. There is a lot of service recommendation methods were expanded in this regard. Reference [1] proposed a novel approach to combine global QoS constraints with dynamic web services selection. Reference [2] 
presented an open, fair and dynamic QoS computation model for web service selection. These QoS-based service selection methods require the user to specify the functional details of the web service and their QoS constraints, but in fact users may not know how to express their demand for services, therefore, these methods is blind and harsh for user [5]. And these methods always assume that the QoS data coming from service providers and users are effective and trustworthy, which is actually impossible in reality and will directly affect the results of web service selection. So the method of web service recommendation that use QoS as the only standard is not perfect. In the reference [10], the authors introduced user comment as the standard of web service composition recommendation method. The appearance of recommended system made a breakthrough, as a widely used technology in the electronic commerce and other areas, it can recommend the users pleased and useful information. Collaborative filtering is the most widely used and the most successful recommendation system. The biggest advantage of it is no need specific content description but the user rating set [6]. Reference [7] presented a trustworthy services selection based on preference recommendation method that assists users in selecting the right web service, according to their own preference. This method can effectively solve the weaknesses of recommendation systems. Thus, this paper based on the above study, proposed a novel web service recommendation method based on credible user comment.

\section{User Evaluation Model}

User Requirements Model. The consumers always have their own requirements when they choose web service. The requirements can be a single target or multiple target. And every target must be provided with constraint. These constraints are a kind of very important parameter that can measure of web service. In addition, the consumer should provide the preference degree of every target.

According to these requirements that are mentioned above, we established a new user requirement model that consists of three elements. Assuming $U_{\mathrm{i}}$ represents the requirement of the $\mathrm{i}^{\text {th }}$ user, we can get $\mathrm{UR}_{\mathrm{i}}=\left\{\left(\mathrm{V}_{\mathrm{j}}, \mathrm{W}_{\mathrm{j}}, \mathrm{T}_{\mathrm{j}}\right), 0<\mathrm{j}<\mathrm{N}\right\}$, where $\mathrm{V}_{\mathrm{j}}$ represents the expected value of the $\mathrm{j}^{\text {th }}$ target, $\mathrm{W}_{\mathrm{j}}$ represents the weight value of the $j^{\text {th }}$ target, $T_{j}$ represents the target type. The user requirement target can be divided into two types, one is the recordable type, and the other one is the unrecordable type. The recordable type is characterized in that the numerical value can be counted by UDDI. It is recorded in execution log. The unrecordable type is characterized in that the numerical value cannot be gotten during execution.

Web Service Model. WS denotes abstract web service that includes four elements such as WS $=<$ Input, Output, Precondition, Results $>$. WS represents one kind of web services which have similar function interface but different effect. There is a set of web service instances for each abstract service type on network. In order to obtain the user satisfaction degree, we need to record the results of web service after every execution. ER denotes the execution result of web service that includes the real value of the recordable type target.

User Evaluation Model. The traditional user evaluation model is always global and convenient that consists of single user evaluation. It reflects overall impression of web service. But the global user evaluation is great ambiguity. It cannot fully reflect the user experience. So sometimes the user evaluation model consists of the local user evaluation. The local model is a good complement to the global model, which evaluates web service from several aspects. So the local model is comprehensive. But now the evaluated index of local model is basically provided by service provider or third parties according to their own professional. In fact some indicators are not concerned by the consumer, or the professional degree of consumer is not enough to give an accurate evaluation. Therefore in this paper we established a new user evaluation model according to user requirements. These evaluation indexes are modeled using fuzzy sets.

The user evaluation can be presented by a number in the range $[0,1]$. That is the basic of the fuzzy representation. Thus in the Eq. 1, user evaluation of web service WS is represented as a fuzzy set UE ${ }_{W S}$ : 


$$
\mathrm{UE}_{\mathrm{wS}}=\left\{u_{k}, A\left(u_{k}\right) \mid u_{k} \in U I\right\}
$$

In Eq. 1, $\mathrm{A}\left(\mathrm{u}_{\mathrm{k}}\right)$ represents the grade of membership of u user requirement index from consumer $\mathrm{k}$. UI represents the set of user requirement index. The membership function of a fuzzy set is a function of ratings that represent user evaluation of web service. We define the membership function for $u_{k}$ in a fuzzy set as Eq. 2:

$$
A\left(u_{k}\right)=\left\{\begin{array}{lr}
0, & 0 \leq u_{k} \leq \alpha \\
{\left[1+\left(\frac{u_{k}-\alpha}{\beta}\right)^{2}\right]^{-1}} & , \alpha<u_{k} \leq 100
\end{array}\right.
$$

\section{A Novel Recommendation Approach Based Credible User Evaluation}

According user requirement model, we can get user expected value $\mathrm{V}_{\mathrm{j}}$. In this paper we use it to compare with the real value $E L_{j}$ which is recorded in execution log to calculate the user satisfaction degree. And then we compare it with the user evaluation for obtaining user trust degree.

User Expected Value Set. User requirement model contains several requirement indexes, and the consumer provided the corresponding constraint condition for every index. But the expression of every index is different, so we need to normalize these indexes. The expression of user requirement indexes can be divided into the following two types. One is the value type. And the other one is the interval type. In order to compare to the real value facilitate, we need to transform the interval type to the value type. At present, there is a variety of deterministic methods. In this paper we use the expanded ordered weighted averaging operator as determining the mathematical formula.

If $\mathrm{f}\left(\mathrm{x}_{1}, \mathrm{x}_{2}, \ldots, \mathrm{x}_{\mathrm{n}}\right)=\Sigma \omega_{\mathrm{i}} \mathrm{y}_{\mathrm{i}}$, where in $\mathrm{W}=\left(\omega_{1}, \omega_{2}, \ldots, \omega_{\mathrm{n}}\right)^{\mathrm{T}}$ is weighted vector which is associated with $f$ and $\omega_{j} \in[0,1], \Sigma \omega_{j}=1, y_{j}$ is the $j^{\text {th }}$ big value in a group of data $\left(x_{1}, x_{2}, \ldots, x_{n}\right)$, the $f(x)$ is called the $n$-dimensional ordered weighted averaging operator.

Assume that $\mathrm{x}=\left\{\mathrm{x} \mid \mathrm{X}_{\mathrm{L}} \leq \mathrm{x} \leq \mathrm{X}_{\mathrm{H}}\right\}$, that means $\mathrm{x}$ is an interval value. The $\mathrm{f}(\mathrm{x})$ function is $\mathrm{Eq}$. 3 :

$$
f_{i}\left(X_{L}, X_{H}\right)=\frac{X_{L}+\lambda X_{H}}{\lambda+1}
$$

We transform the user evaluation of the interval type to the value type through the Eq. 4:

$$
E V_{i}\left(X_{L}, X_{H}\right)=f_{i}\left(X_{L}, X_{H}\right)= \begin{cases}X_{H}, & \lambda \rightarrow 0 \\ \frac{X_{L}+X_{H}}{2}, & \lambda \rightarrow 1 \\ X_{L}, & \lambda \rightarrow \infty\end{cases}
$$

In Eq. 4, i represent the index number of user requirements. After normalized user requirement set, we can get the user expected value set EV.

User Satisfaction Degree. Then we need to calculate the distance between the real value of execution log and the user expected value. We use the gray correlation analysis method to calculate the user satisfaction degree. Assumed that $r\left(E_{j}, E L_{j}\right)$ represents the correlation value between the user expected value and real value of operation. So we can get the Eq. 5 as follows:

$$
\begin{aligned}
& d(j)=\left|E V_{j}-E L_{j}\right| \\
& \mathrm{SD}(j)=r\left(E V_{j}, E L_{j}\right)=\frac{\varepsilon d_{\max }}{d(j)+\varepsilon d_{\text {max }}}
\end{aligned}
$$

Where $\varepsilon \epsilon[0,1]$ represents the resolution value. $\mathrm{SD}(\mathrm{j})$ represents the user satisfaction degree of the $\mathrm{j}^{\text {th }}$ recordable user requirement index.

Trust Degree of User Evaluation Calculation. In the section, we compare the user evaluation value with user satisfaction degree of customer $\mathrm{k}$. The distance of two values is closer, the trust degree of the user evaluation is higher. Assumed $\mathrm{TD}_{\mathrm{k}}$ represents the trust degree of user evaluation of the customer $\mathrm{k}$. We can get the Eq. 6: 


$$
\begin{aligned}
& T D_{k}(j)=1-\left|\mathrm{SD}_{k}(j)-A\left(u_{k}(j)\right)\right| \\
& T D_{k}=\frac{\sum_{j=1}^{N} T D_{k}(j)}{\mathrm{N}}
\end{aligned}
$$

The Novel Web Service Recommendation Approach. After obtaining the trust degree of each user evaluation, we have to assign the performance ratings to service alternative $\mathrm{WS}_{\mathrm{i}}$ with respect to user requirement $\mathrm{UR}_{\mathrm{j}}$. The fuzzy performance rating matrix $\mathrm{R}$ is shown as:

$$
\mathrm{R}=\left[r_{i j}\right]_{m \times n}=\begin{aligned}
& W S_{1} \\
& W S_{2} \\
& \vdots \\
& W S_{m}
\end{aligned}\left[\begin{array}{cccc}
r_{11} & r_{12} & \cdots & r_{1 n} \\
r_{21} & r_{22} & \cdots & r_{2 n} \\
\vdots & \vdots & \vdots & \vdots \\
r_{m 1} & r_{m 2} & \cdots & r_{m n}
\end{array}\right]
$$

Where $\mathrm{r}_{\mathrm{ij}}$ represents the individual fuzzy rating of web service $\mathrm{WS}_{\mathrm{i}}$ with respect to attribute $\mathrm{UR}_{\mathrm{j}}$.the trusted user evaluation-based rating is derived by the additive rule as follows:

$$
r_{i j}=\frac{\sum_{k=1}^{m}\left(T D_{k} * \mu_{k}(i j)\right)}{\sum_{k=1}^{m} T D_{k}}
$$

Where $\mathrm{TD}_{\mathrm{k}}$ indicates the consumer's trust degree that measured by Eq. 6 .

$$
\begin{aligned}
& P\left(W S_{i}\right)=\left[r_{i j}\right] \otimes\left[\omega_{j}\right]^{\mathrm{T}}=\left[\begin{array}{c}
\mathrm{P}\left(W S_{1}\right) \\
\mathrm{P}\left(W S_{2}\right) \\
\vdots \\
\mathrm{P}\left(W S_{n}\right)
\end{array}\right] \\
& =\left[\begin{array}{cccc}
r_{11} & r_{12} & \cdots & r_{1 n} \\
r_{21} & r_{22} & \cdots & r_{2 n} \\
\vdots & \vdots & \vdots & \vdots \\
r_{m 1} & r_{m 2} & \cdots & r_{m n}
\end{array}\right] \otimes\left[\begin{array}{c}
\omega_{1} \\
\omega_{2} \\
\vdots \\
\omega_{n}
\end{array}\right] \\
& =\left[\begin{array}{c}
\left(r_{11} \otimes \omega_{1}\right) \oplus\left(r_{12} \otimes \omega_{2}\right) \oplus \cdots \oplus\left(r_{1 n} \otimes \omega_{n}\right) \\
\left(r_{21} \otimes \omega_{1}\right) \oplus\left(r_{22} \otimes \omega_{2}\right) \oplus \cdots \oplus\left(r_{2 n} \otimes \omega_{n}\right) \\
\vdots \\
\left(r_{m 1} \otimes \omega_{1}\right) \oplus\left(r_{m 2} \otimes \omega_{2}\right) \oplus \cdots \oplus\left(r_{m n} \otimes \omega_{n}\right)
\end{array}\right]
\end{aligned}
$$

Since service customers have their different subjective perception on user requirement target, it needs to define an index of priority on candidate service. In the context, web service priority (denoted as $\mathrm{P}$ ) is defined to specify the consumer perception on web services $\mathrm{WS}_{\mathrm{i}}$. It can be formulated by the inference rule using fuzzy simple additive weighting rule as Eq. 9.

\section{A Case Study}

In this section we design an experiment to prove the efficiency of the novel web service recommendation method that is based the credible user evaluation. Assume that we have four candidate web services which have similar function. And there are six consumers who use every web service several times. Firstly we give their requirement indexes which are presented in Table 1. 
Table 1. User requirement indexes.

\begin{tabular}{|c|c|c|c|c|c|}
\hline Indexes & Cost & Time & Through & Reliability & Available \\
\hline User1 & 8 & $0.3 \mathrm{~s}$ & $>80$ & $>90$ & $>95$ \\
(Weight) & $(0.1)$ & $(0.3)$ & $(0.1)$ & $(0.3)$ & $(0.2)$ \\
\hline User2 & $<10$ & $<0.6 \mathrm{~s}$ & 88 & 93 & 90 \\
(Weight) & $(0.2)$ & $(0.4)$ & $(0.2)$ & $(0.1)$ & $(0.1)$ \\
\hline User3 & 7 & 0.6 & 92 & $>95$ & $>90$ \\
(Weight) & $(0.2)$ & $(0.1)$ & $(0.3)$ & $(0.2)$ & $(0.2)$ \\
\hline User4 & {$[8,15]$} & $0.8 \mathrm{~s}$ & {$[80,90]$} & {$[90,95]$} & $>98$ \\
(Weight) & $(0.1)$ & $(0.2)$ & $(0.2)$ & $(0.2)$ & $(0.3)$ \\
\hline User5 & 6 & {$[0.2,0.8]$} & 90 & 88 & 85 \\
(Weight) & $(0.2)$ & $(0.2)$ & $(0.2)$ & $(0.2)$ & $(0.2)$ \\
\hline User6 & 10 & $0.10 \mathrm{~s}$ & $>70$ & 80 & 80 \\
(Weight) & $(0.1)$ & $(0.3)$ & $(0.3)$ & $(0.1)$ & $(0.2)$ \\
\hline
\end{tabular}

Through Table 1 we can see there are six consumers who use every web service times. The requirement indexes are presented as different way. We use Eq. 3 and Eq. 4 to quantize requirement indexes. Table 2 presents the execution log of four candidate web services. We compare it to the expected values which are calculated from Table 1. We use Eq. 5 to get the user satisfaction degrees which are presented in Table 3.

Table 2. The average real value of execution log.

\begin{tabular}{|c|c|c|c|}
\hline WS & Cost & Time & Through \\
\hline WS1 & 9 & 0.7 & 80 \\
\hline WS2 & 8.5 & 0.5 & 85 \\
\hline WS3 & 8 & 0.6 & 95 \\
\hline WS4 & 10 & 0.4 & 90 \\
\hline
\end{tabular}

Table 3. The satisfaction degree of consumer.

\begin{tabular}{|c|c|c|c|c|c|c|c|}
\hline WS & Index & User1 & User2 & User3 & User4 & User5 & User6 \\
\hline \multirow{4}{*}{ WS1 } & Cost & 0.62 & 0.47 & 0.55 & 0.64 & 0.52 & 0.62 \\
\cline { 2 - 8 } & Time & 0.45 & 0.44 & 0.62 & 0.76 & 0.44 & 0.44 \\
\cline { 2 - 8 } & Through & 0.48 & 0.44 & 0.44 & 0.62 & 0.44 & 0.44 \\
\hline \multirow{3}{*}{ WS2 } & Cost & 0.80 & 0.49 & 0.63 & 0.59 & 0.56 & 0.52 \\
\cline { 2 - 8 } & Time & 0.62 & 0.49 & 0.62 & 0.52 & 1.0 & 0.55 \\
\cline { 2 - 8 } & Through & 0.55 & 0.70 & 0.58 & 1.0 & 0.62 & 0.52 \\
\hline \multirow{3}{*}{ WS3 } & Cost & 1.0 & 0.50 & 0.72 & 0.56 & 0.62 & 0.44 \\
\cline { 2 - 8 } & Time & 0.52 & 0.44 & 1.0 & 0.62 & 0.62 & 0.50 \\
\cline { 2 - 8 } & Through & 0.78 & 0.48 & 0.70 & 0.44 & 0.77 & 0.76 \\
\hline \multirow{3}{*}{ WS4 } & Cost & 0.45 & 0.44 & 0.44 & 0.75 & 0.44 & 1.0 \\
\cline { 2 - 8 } & Time & 0.80 & 0.55 & 0.44 & 0.44 & 0.62 & 0.62 \\
\hline
\end{tabular}




\begin{tabular}{|l|l|l|l|l|l|l|l|}
\hline & Through & 0.62 & 0.77 & 0.83 & 0.62 & 1.0 & 0.62 \\
\hline
\end{tabular}

Table 4 presents the user comment that is evaluated by fuzzy set. Assumed that $\alpha=30, \beta=20$, We use Eq. 2 to get the degree of membership. Then we use Eq. 6 to calculate the trust degree of user evaluation. The trust degree is presented in Table 5.

Table 4. User comment.

\begin{tabular}{|c|c|c|c|c|c|c|c|}
\hline WS & Index & User1 & User2 & User3 & User4 & User5 & User6 \\
\hline \multirow{5}{*}{ WS1 } & Cost & 60 & 40 & 60 & 70 & 50 & 80 \\
\cline { 2 - 8 } & Time & 50 & 40 & 60 & 80 & 50 & 80 \\
\cline { 2 - 8 } & Through & 50 & 40 & 50 & 60 & 50 & 80 \\
\cline { 2 - 8 } & Reliability & 70 & 60 & 60 & 80 & 60 & 80 \\
\cline { 2 - 8 } & Available & 60 & 60 & 60 & 80 & 60 & 80 \\
\hline \multirow{5}{*}{ WS2 } & Cost & 90 & 40 & 60 & 60 & 60 & 80 \\
\cline { 2 - 8 } & Time & 70 & 40 & 60 & 50 & 90 & 70 \\
\cline { 2 - 8 } & Through & 50 & 40 & 60 & 90 & 60 & 70 \\
\cline { 2 - 8 } & Reliability & 80 & 60 & 60 & 60 & 60 & 70 \\
\hline \multirow{5}{*}{ Wv3 } & Available & 80 & 60 & 60 & 60 & 50 & 70 \\
\cline { 2 - 8 } & Cost & 95 & 40 & 70 & 60 & 70 & 70 \\
\cline { 2 - 8 } & Through & 80 & 40 & 80 & 50 & 90 & 70 \\
\cline { 2 - 8 } & Reliability & 80 & 40 & 70 & 70 & 70 & 70 \\
\cline { 2 - 8 } & available & 80 & 40 & 70 & 70 & 70 & 70 \\
\hline \multirow{5}{*}{ WS4 } & Cost & 50 & 40 & 50 & 80 & 50 & 90 \\
\cline { 2 - 8 } & Time & 80 & 50 & 50 & 50 & 60 & 90 \\
\cline { 2 - 8 } & Through & 60 & 60 & 60 & 60 & 90 & 90 \\
\cline { 2 - 8 } & Reliability & 60 & 60 & 70 & 70 & 70 & 90 \\
\hline
\end{tabular}

Table 5. The trust degree of user evaluation.

\begin{tabular}{|c|c|c|c|c|c|c|}
\hline User & User1 & User2 & User3 & User4 & User5 & User6 \\
\hline TD & 0.84 & 0.65 & 0.82 & 0.85 & 0.82 & 0.73 \\
\hline
\end{tabular}

Through the result of Table 5, we can know obviously that the trust degree of User2 and User6 is lower. That means the user evaluation of User2 and User6 is uncrediable.

Table 6. Experiment result.

\begin{tabular}{|c|c|c|c|c|}
\hline WS & WS1 & WS2 & WS3 & WS4 \\
\hline Approach1 & 0.9 & 0.8 & 0.68 & 0.7 \\
\hline Approach2 & 0.8 & 0.7 & 0.88 & 0.6 \\
\hline
\end{tabular}

Table 6 shows the experiment result. If we use trandional web service recommendation approach without trust degree,which is called Approach1 from Table 6, we can get WS1 $>$ WS2 $>$ WS4 $>$ WS3. But In fact WS1 is not the best candidate web service composition. We use novel recommendation approach which is called Approach2 to get WS3 $>$ WS1 $>$ WS2 $>$ WS4. That is the correct soluation. So 
we can see the novel approach of web service composition recommendation can avoid the reflaction of uncrediable user evaluation.

\section{Summary}

In this paper, the content of the research is to apply credible user evaluation to web service recommendation method. According to the user requirement model, this paper established user evaluation model which consist of recordable type and un-recordable type. And then according to the real value of execution log, we use gray correlation algorithm to calculate the consumer satisfaction degree. Comparing the user evaluation with consumer satisfaction degree, we obtain the trust degree of user evaluation. Then we used fuzzy analysis method to get the best candidate web service. Finally, through one case we prove the new method can effectively avoid the influence of exaggerated and false user evaluation.

\section{Acknowledgment}

Denghui Wang would like to extend sincere gratitude to corresponding author, Hao Huang, for his instructive advice and useful suggestions on this research. And we thank the anonymous reviewers for their valuable feedback and suggestions.

\section{References}

[1] Zheng Yanwei, Deng Haojiang and Liu Lei: A dynamic web services selection based on decomposition of global QoS constraints. IEEE Youth Conference on Information Computing and Telecommunications (YC-ICT) (2010), p.77-80

[2] Gao Yan, Zhang Bin and Shi Shaowei: A User Requirement-Driven Service Dynamic Personalized QoS Model. The Third International Conference on Dependability (2010), p. 17-23

[3] Y. Wang, J. Vassileva: A review on trust and reputation for Web service selection. Proc. 27th International Conference on Distrbuted Computing Systems Workshops (2007), p.25-32

[4] Wang Ping, Chao Kuoming and Lo Chichun: An evidence-based scheme for web service selection. Information Technology and Management Vol.12 (2011), p.161-172

[5] Li Yan, Zhou Minghui and Li Ruichao: Service selection Approach Considering the Trustworthiness of QoS Data. Journal of software Vol.19 (2008), p.2620-2627

[6] Xu HL, Wu X and Li XD: Comparison study of Internet recommendation system. Journal of Software Vol.20 (2009), p.350-362

[7] Zhu Rui, Wang Huaimin and Feng Dawei: Trustwothy services selection based on preference recommendation. Journal of Software Vol.22 (2011), p.853-864

[8] Gong Songjie, Ye Hongwu and Tan Hengsong: Combining Memory-based and Model-Based Collaborative Filtering in Recommender Systme. PACCS '09. Pacific-Asia Conference on Communications and Systems (2009), p.690-693

[9] Zhou Ning, Xie Junyuan: Select web services based on qualitative multi-users preferences. Acta Electronica Sinica Vol.39 (2011), p.729-736

[10]Cui Yuhui, Chen Chang and Zhao Zhengde: Web service Selection Based on Credible User Recommended and QoS. IEEE/ACIS 11th International Conference on Computer and Information Science (2012), p.637-642 